\title{
Un modèle de cofinancement de la formation continue orienté vers l'usager: le cas espagnol
}

\section{Isabel Voirol-Rubido}

Cet article s'intéresse aux effets d'un dispositif de financement de la formation continue orienté vers le demandeur de formation. Il analyse la qualité du dispositif espagnol en termes d'efficacité, d'équité d'accès et d'incitation des actifs occupés espagnols à s'engager en formation. En s'appuyant sur une analyse de données secondaires combinant une méthodologie de natures quantitative et qualitative, il met en évidence son potentiel à améliorer l'accès à la FC des salariés espagnols entre 2004 et 2011. Il relève cependant la difficulté du dispositif à contrecarrer l'effet d'instrumentalisation de la formation par les employeurs et à déjouer l'impact du coût d'opportunité sur la décision d'investir en formation pénalisant ainsi l'investissement en formation continue.

\section{Introduction}

Les capacités financières individuelles et la volonté ou non de l'employeur de soutenir l'effort formation de ses collaborateurs ont une influence évidente sur le potentiel d'accès à la formation continue (CEDEFOP, 2015; Lambert \& Marion-Vernoux, 2014).

L'entreprise sélectionne les salariés qui présentent à ses yeux les meilleures chances de rentabiliser son investissement à qui elle impose ses choix de formation (nature, modalités, profils de salariés) en fonction de ses besoins spécifiques. Elle détient ainsi un pouvoir d'instrumentalisation de la formation contre lequel le salarié est impuissant ce qui contribue à créer des inégalités d'accès à la formation continue (Dubois \& Melnik-Olive, 2017).

Face à ces constats, les regards se tournent désormais vers un système de financement public de la formation continue orienté vers le demandeur de formation auquel on prête un potentiel à inciter les individus à se former (Backes-Gellner, 2011) indépendamment de la volonté de l'employeur. Deux exemples helvétiques illustrent ce glissement. Le canton de Berne a, par exemple, expérimenté positivement le potentiel des bons de formation à inciter les actifs occupés 
auxquels ces bons ont été proposés à se former (Wolter \& Messer, 2009). Le canton de Genève a quant à lui introduit avec un certain succès le chèque annuel de formation qui octroie, sous certaines conditions, un financement public aux actifs occupés pour se former (Cour des comptes de Genève, 2015).

Par ailleurs, le principe du cofinancement consistant à répartir les coûts sur tous les bénéficiaires de la formation semble remporter l'adhésion et est particulièrement encouragé par les organisations internationales telles que l'OCDE (2004) pour rendre l'investissement économiquement soutenable.

Cependant, une étude récente révèle la réticence des partenaires sociaux suisses à envisager ce type de modalités pour la Suisse actuellement (VoirolRubido, 2017). Les principales craintes évoquées sont l'inefficacité économique du modèle liée au risque de substitution des fonds privés par des fonds publics ainsi qu'à la lourdeur administrative qu'il engendrerait. Cette dernière compromet à leurs yeux la flexibilité et la réactivité de l'offre de formation dont l'économie a besoin. De plus, l'orientation du financement vers la demande leur semble inadaptée aux publics peu qualifiés qui sont justement ceux qui peinent particulièrement à se former. Finalement, une ingérence de l'État dans la gestion entrepreneuriale est fortement redoutée.

Mais ces craintes sont-elles fondées? Un cofinancement tripartite de la formation continue orienté vers la demande est-il en mesure d'améliorer efficacement l'accès à la formation continue notamment celui des salariés peu qualifiés?

Nos regards se tournent alors vers l'Espagne qui a mis en place un modèle de financement de la formation continue orienté vers la demande soutenu par les pouvoirs publics, les entreprises et les salariés. Il repose sur plusieurs volets. Celui qui s'adresse aux actifs occupés, désigné de dispositif de demande de formation, fait l'objet de notre analyse.

Cet article rend compte de la potentialité de ce dispositif à améliorer la qualité d'un système de formation continue en termes d'efficacité, d'équité d'accès et d'incitation des actifs occupés à s'engager en formation. Il présente dans un premier temps le cadre conceptuel traitant de la problématique de l'accès à la formation continue et de son financement. Il définit ensuite la question et les hypothèses de recherche et décrit le dispositif de recherche déployé. Sont ensuite évoqués et discutés les principaux résultats pour finalement conclure.

\section{L'investissement en formation continue et son financement}

Les firmes privilégient les activités de formation en faveur des collaborateurs les plus à même de maximiser la rentabilisation économique de l'investissement induisant ainsi une inégalité d'accès à la formation continue (Dubois \& MelnikOlive, 2017). Il apparaît, en effet, que cette dernière profite avant tout aux jeunes actifs occupés à des postes à responsabilité de préférence dans une grande 
entreprise (Chéron, 2011; Hanhart, 2007) au détriment des salariés peu ou pas qualifiés, des seniors et de ceux insérés dans le marché du travail secondaire qui regroupe les emplois précaires et instables.

La rentabilité de l'investissement sera d'autant maximisée que la formation sera spécifique, c'est-à-dire qu'elle répondra aux besoins de la fonction et du poste du formé, s'accordant ainsi aux mécanismes mis en évidence par la Théorie du capital humain (Becker, 1964). Cette dernière non seulement met en relation la productivité de l'individu avec son niveau de formation mais conditionne l'augmentation de productivité du salarié à la pertinence des compétences acquises lors de la formation au regard du poste occupé. Rentabilité d'autant améliorée si la mobilité du collaborateur est réduite et si l'augmentation de la productivité de ce dernier est plus forte dans l'entreprise qui le forme que dans d'autres firmes (Pichler, 1993). Cette pratique d'instrumentalisation de la formation, contre laquelle le salarié est impuissant, est bien ancrée en milieu professionnel malgré les études démontrant l'effet positif de la formation générale sur la productivité des firmes et, par voie de conséquence, sur la croissance économique (Hansson, 2008). À noter également que la rentabilité d'une formation est influencée par les coûts directs et indirects (coûts d'opportunité) qu'elle engendre. Dans la perspective patronale, l'absence du collaborateur en formation péjore la productivité de l'entreprise induisant un coût indirect qui se traduit par un manque à gagner ce qui influence la décision d'investir ou non en formation (Hanhart, 2007).

Lorsque les firmes ne souhaitent pas financer les activités de formation de leurs collaborateurs et qu'aucune obligation légale généralisée n'existe en la matière, ce qui est le cas, par exemple, en Suisse, ce financement repose alors sur les seules épaules de l'individu souhaitant se former. La personne est alors confrontée à deux problèmes majeurs: celui d'assumer les coûts de sa formation et celui de trouver la disponibilité pour se former. Dans les deux cas, la question du financement s'impose.

Les bénéfices de la formation n'étant jamais ni totalement privés ni totalement publics, une répartition de son financement entre ses différents bénéficiaires, à savoir, l'individu, l'entreprise et la collectivité publique semble justifiée. On parle alors de cofinancer l'investissement en formation (OCDE, 2004).

Par ailleurs, les expériences faites à ce jour en matière de financement de la formation continue orienté vers la demande laissent présager des résultats intéressants au regard de leur potentiel à améliorer l'accès à la formation continue (Backes-Gellner, 2011; CSRE, 2004). Ce type de financement influencerait en principe positivement la motivation des individus à s'engager en formation qui disposent ainsi d'un pouvoir d'achat supplémentaire (CSRE, 2004). Il serait de plus à mettre à son crédit une probable amélioration de l'efficience des allocations de formation du fait que les prestataires de formation seraient davantage contraints de proposer des offres qui répondent aux besoins spécifiques des individus (CSRE, 2004; OCDE, 2004). 


\section{Questions et hypothèses de recherche}

Notre analyse s'intéresse à la qualité du dispositif de demande de formation mis en place en Espagne. En d'autres termes, ce dispositif a-t-il incité les actifs occupés espagnols à s'engager en formation? A-t-il contribué à augmenter le nombre de participants à la formation continue depuis sa mise en oeuvre? Et, l'accès à la formation continue est-il plus équitable?

Ces questions s'appuient sur les hypothèses de recherche suivantes. La première suppose que le modèle espagnol permette de se prémunir contre l'influence négative du coût d'opportunité sur l'accès à la formation continue puisque le dispositif de financement couvre la perte de gain encourue à la fois par l'entreprise formatrice et le formé. Il devrait par ailleurs être en mesure d'éviter, ne serait-ce que partiellement, l'instrumentalisation de la formation par les employeurs puisque le formé, cofinanceur de sa formation, détient un pouvoir décisionnel le rendant responsable de son parcours de formation. De plus, en tant qu'instrument de financement orienté vers la demande, il devrait avoir un effet incitatif sur l'individu à s'engager en formation. Enfin, le principe du cofinancement inciterait l'employeur à engager tout profil de collaborateurs en formation en raison de la répartition du coût de formation sur tous ses bénéficiaires, en l'occurrence les employeurs, les salariés et les pouvoirs publics.

\section{La démarche méthodologique}

L'analyse de la potentialité du dispositif espagnol à améliorer l'accès à la formation continue des actifs occupés a nécessité, dans un premier temps, une analyse de contenu basée sur les textes législatifs le concernant, spécifiés au point ci-dessous.

Elle procède ensuite essentiellement d'une analyse statistique descriptive, entièrement réalisée par nos soins, ayant pour objectif de décrire et dessiner des tendances. Des indicateurs ont ainsi été élaborés pour tenter de cerner l'efficacité, l'équité d'accès et le potentiel à susciter l'engagement en formation du modèle espagnol. Une attention particulière a été portée sur la situation des publics les plus lésés dans l'accès à la formation continue, à savoir, les personnes peu qualifiées, les seniors de plus de quarante-cinq ans et les actifs occupés dans le marché du travail secondaire.

L'analyse quantitative secondaire mobilise des données statistiques publiques agrégées fournies par la FTFE (Fundación Tripartita para la Formación en el Empleo), organe public de gestion de la formation continue en Espagne. Elle s'appuie également sur des données statistiques fournies par l'INE (Institut national de statistiques espagnol) qui, par le biais de ces enquêtes sur la population active (EPA) et son Directoire central des entreprises espagnoles (DIRCE), fournit des données utiles pour l'analyse et l'interprétation des résultats. 
L'analyse quantitative secondaire des données a été complétée par une analyse qualitative du dispositif de demande de formation résultant des données récoltées dans le cadre d'une évaluation externe du dispositif espagnol portant sur les années 2007-2008, réalisée par les équipes GAPEFet GIPE sous mandat de la FTFE (FTFE, 2010). Ces équipes ont récolté l'information moyennant des entretiens non directifs, des groupes de discussion et la mobilisation d'experts de la formation continue en Espagne et de représentants d'entités organisatrices de formation selon la méthode DELPHI. Cette partie du rapport a eu pour objectif de déterminer l'efficacité, l'efficience et l'impact du dispositif de demande de formation.

L'articulation entre notre analyse quantitative et l'analyse qualitative réalisée par les équipes GAPEF et GIPE a été possible du fait que toutes deux concernent le même objet d'étude et mobilisent la même base de données fournie par la FTFE. Les résultats du rapport GAPEF-GIPE ne sont toutefois évoqués dans notre travail que sporadiquement lorsqu'ils fournissent des éléments de compréhension à certains des résultats de notre analyse quantitative.

Le système de formation continue étudié, initialement mis en place en Espagne en 1993, a connu diverses modifications au cours du temps. La période d'observation retenue pour la présente analyse s'étend de 2004 à 2010, voire 2011 pour certains indicateurs, afin de tenir compte d'une organisation constante du dispositif telle que décrite au point suivant.

\section{Le dispositif de demande de formation}

Le dispositif de demande de formation prévoit le droit d'accéder à la formation continue à tout actif occupé que ce soit dans le cadre d'une formation souhaitée par l'entreprise (interne ou externe) ou dans celui d'un projet personnel de formation certifiée (permis individuel de formation - PIF), que la formation soit spécifique ou générale. À cela s'ajoutent des actions d'accompagnement à la formation et un devoir d'information et de défense du droit à la formation des salariés assigné à leurs représentants légaux.

Ces mesures sont cofinancées par les pouvoirs publics (nationaux, régionaux et européens), les entreprises et les salariés. L'employeur et ses collaborateurs versent respectivement $0,6 \%$ et $0,1 \%$ de la masse salariale de l'entreprise à un fonds spécifique géré par la sécurité sociale. Les firmes de plus de neuf salariés ont par ailleurs l'obligation de financer une partie des coûts directs de la formation liée à leurs besoins, soit entre $10 \%$ et $40 \%$ en fonction de la taille de l'entité. Plus la taille est grande plus la part du cofinancement exigé est important. Cette obligation ne s'applique néanmoins pas dans le cas des PIF.

Le financement public procède d'un système de bonification. Le principe consiste à mettre à disposition du demandeur un crédit de formation d'un volume prédéfini destiné à couvrir les coûts directs et indirects de 
l'investissement. Concrètement et conformément à l'art. 11 al.1 TAS/2307/2007, du 27 juillet, en vigueur durant notre période d'observation (2004-2011), ce crédit de formation correspond au pourcentage de bonification défini dans la loi générale sur le budget de l'État que l'on applique au montant prélevé par l'entreprise au titre de cotisation pour la formation professionnelle l'année précédant l'exercice en cours. Les employeurs ont alors l'obligation de renseigner le représentant des travailleurs et la FTFE de l'action de formation souhaitée (dates, lieu, horaires, etc.) sept jours avant le démarrage du projet pour lequel la bonification est demandée (TAS 2307/2007, art. 18, al. 1). La bonification prend effet à l'issue de la formation (RD 395/2007, art. 17 al. 2) mais au plus tard à la fin de l'année civile en cours (TAS 2307/2007, art. 19 al. 1). L'entreprise déduit à ce moment-là le montant de la bonification des versements qu'elle effectue à la sécurité sociale.

À noter que le pourcentage de bonification est inversement proportionnel à la taille de l'entreprise allant d'une bonification complète pour les entreprises de 6 à 9 salariés jusqu'à $50 \%$ pour les firmes de plus de 250 collaborateurs. Les microentreprises, c'est-à-dire celles de moins de 5 salariés, bénéficient quant à elles d'un crédit de formation annuel de l'ordre de $€ 420$.

\section{L'interprétation et la discussion des résultats}

Notre analyse du dispositif de demande de formation met en évidence les points de réflexion suivants.

\section{L'impact global du dispositif sur l'accès à la formation continue}

Le premier point concerne l'impact global d'un tel dispositif sur l'accès à la formation continue des actifs occupés. Cet impact a été évalué en mobilisant les données statistiques fournies par la FTFE et l'INE.

Dans la perspective temporelle allant de 2004 à 2010, le dispositif espagnol a contribué à augmenter la participation à la formation des actifs occupés espagnols que ce soit en termes de nombre de formés (env. 600'000 en 2004 et env. 2'800'000 en 2010) ou de taux de participation (3\% en $2004-15 \%$ en 2010). Ce dernier a été calculé en rapportant le nombre total de formés par le dispositif au volume de population active occupée, comme l'illustre le graphique suivant. 


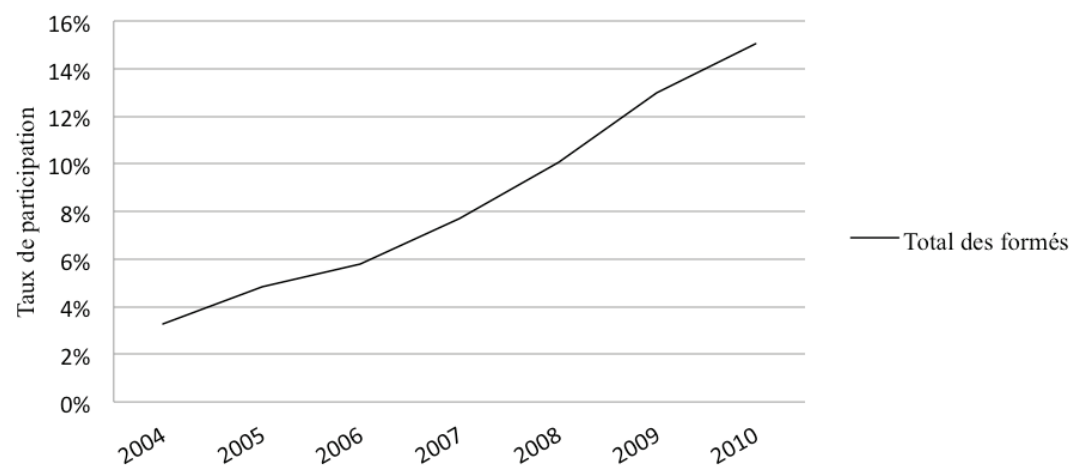

Graphique 1. Taux de participation au dispositif de demande de formation de 2004 à 2010

Source: auteure, calculé sur la base des données statistiques fournies par la FTFE et l'INE

Notons, cependant, que cette évolution s'accompagne d'une baisse du volume d'heures de formation continue annuelles dont bénéficient en moyenne les formés ( $40 \mathrm{~h}$ en 2004 et $35 \mathrm{~h}$. en 2010). Malgré cette baisse de volume d'heures de formation, le dispositif espagnol se révèle efficace pour améliorer l'accès global à la formation continue de la population active occupée, en particulier de 2006 à 2010.

Cet impact positif doit toutefois être nuancé au regard de son évolution dans le temps. On assiste, en effet, à une plus forte augmentation du taux de participation des actifs occupés espagnols entre 2006 (6\%) et 2010 (15\%) qu'entre $2004(5 \%)$ et $2006(6 \%)$. Le manque de réactivité du système de formation continue à répondre aux besoins des demandeurs entre 2004 et 2005 est mis en cause (Pineda-Herrero, 2007; Pineda \& Sarramona, 2006). Des améliorations sont alors apportées. Le dispositif devient plus flexible tant au niveau de sa gestion (amélioration des délais d'obtention du financement, meilleure lisibilité des moyens financiers à disposition, soulagement d'une lourdeur administrative mal perçue par les employeurs et coûteuse pour les différents partenaires) que du type de formation éligible pour l'obtention du financement. Mais la forte augmentation du taux de participation constatée entre 2006 (6\%) et $2010(15 \%)$ a sans doute également été influencée par le contexte de ralentissement économique auquel doit faire face l'Espagne à cette période. Il est, en effet, à supposer que certaines entreprises cherchent à rester compétitives en mobilisant la formation pour développer les compétences de leurs collaborateurs qui disposent de temps pour se former lorsque les cadences de production se réduisent. Démarche favorisée par une mobilité professionnelle externe des travailleurs, réduite en raison des tensions sur le marché du travail. 
La deuxième nuance qu'il convient d'émettre concerne le potentiel du dispositif à lisser les inégalités d'accès à la formation continue, illustré dans le graphique qui suit.

Proportion des publics prioritaires par rapport à l'ensemble de la population active occupée et proportion des publics prioritaires formés par le dispositif de demande de formation par rapport à l'ensemble des formés par ce dispositif, de 2004 à 2010

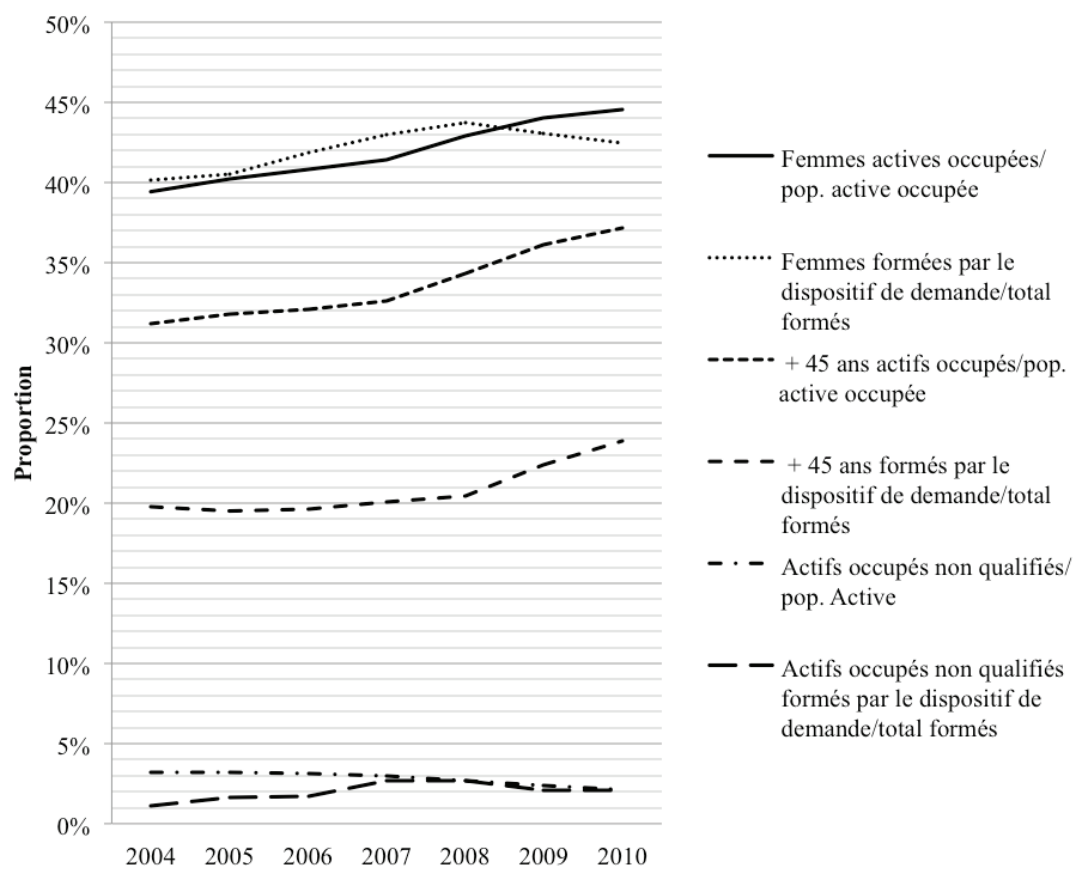

Graphique 2. Taux de participation des publics prioritaires

Source: auteure, calculé sur la base des données statistiques fournies par la FTFE et l'INE

Le niveau de formation, l'âge, les caractéristiques du poste occupé ou la taille de l'entreprise restent des déterminants non négligeables dans l'accès à la formation continue. Phénomènes qui s'observent de manière récurrente dans tous les contextes (voir par exemple Bernier, 2014, pour le contexte canadien).

Ainsi, la formation en entreprise continue de profiter avant tout aux personnes les mieux formées. Ce phénomène n'est pas surprenant dans le contexte espagnol qui se caractérise par un recours accru à la formation continue pour ajuster les compétences des jeunes diplômés à celles requises par le marché du travail (Dautray, 2007). Fait réjouissant, toutefois, au fil des années observées, le taux d'accès à la formation continue des actifs occupés sans formation s'améliore pour 
atteindre une proportion similaire au taux de représentation de cette cohorte dans la population active occupée $(2,1 \%)$.

S'agissant de l'impact de l'âge sur l'accès à la formation continue, l'analyse du dispositif de demande de formation fait ressortir une sous-représentation de plus en plus marquée des actifs occupés de plus de quarante-cinq ans dans la formation continue. En effet, ils représentaient 31\% de la population active occupée mais seulement près de $20 \%$ des formés en 2004 (écart de $11 \%$ ). Cet écart s'accentue légèrement en 2010 puisque cette cohorte représentait alors 37\% de la population active occupée et seulement $24 \%$ des formés (écart de 13\%).

Nous constatons par ailleurs que jusqu'en 2008, les femmes participent au dispositif de demande de formation dans une proportion légèrement plus forte que leur représentation dans le tissu économique espagnol. Une tendance vers la sous-représentabilité des femmes dans la formation continue organisée par les entreprises se dessine néanmoins dès 2009. En effet, alors que la proportion de femmes actives occupées par rapport à l'ensemble de la population active occupée de plus de 16 ans connaît une augmentation constante de 2004 à 2008, passant d'environ $40 \%$ à près de $44 \%$, la proportion de femmes formées par le dispositif de demande de formation amorce une baisse dès 2009 (43\% en 2009, 42\% en 2010) bien que leur implication dans le marché de l'emploi continue d'augmenter. En cause, sans doute, des raisons exogènes au dispositif davantage liées aux caractéristiques du marché du travail qu’à celles du dispositif de formation. Les femmes occupent, en effet, davantage d'emplois dont les caractéristiques (temps partiel, CDD, secteur d'activité, etc.) pénalisent l'accès à la formation continue, précarité sans doute exacerbée en période de crise économique.

Par ailleurs, dans la grande majorité des cas, les formés travaillent dans de grandes firmes (plus de 250 salariés) (près de $70 \%$ en 2004 et près de $50 \%$ en 2010). Cependant, une tendance vers une plus forte implication des salariés des petites entreprises et une plus faible implication des salariés des grosses structures se profilent. Les mieux lotis étant les salariés des entreprises de moins de 50 salariés, qui voient leur taux de participation passer d'environ $12 \%$ en 2004 à près de $30 \%$ en 2010 . L'évolution du taux de participation des salariés des microentreprises de moins de 10 collaborateurs est également marquante puisqu'il passe d'environ $2 \%$ en 2004 à près de $12 \%$ en 2010 . Néanmoins, cette évolution révèle sans doute un glissement du tissu économique espagnol vers les PME et les microentreprises, en particulier celles n'occupant pas de salariés, suite à la crise économique précédemment évoquée.

\section{L'impact du dispositif sur les entreprises espagnoles}

Le deuxième point de discussion déplace la réflexion de la perspective du salarié vers celle des employeurs en s'intéressant à l'impact du dispositif sur les entreprises espagnoles. Cette focale d'analyse est importante dans la mesure où le rôle de l'employeur dans l'accès à la formation des salariés est primordial, comme le démontre notamment le nouveau dispositif d'enquêtes français sur les formations 
et itinéraires des salariés (Defis) permettant de cerner le recours à la formation des entreprises privées (Dubois, Marion-Vernoux \& Noack, 2016).

L'amélioration de l'impact du dispositif de demande de formation sur les entreprises se constate tout d'abord par l'augmentation constante du nombre total d'entreprises qui s'y sont enregistrées entre 2004 et 2010 et par la proportion en augmentation de collaborateurs engagés dans des activités de formation continue. Augmentation qui s'observe d'ailleurs surtout dans les PME de moins de 10 salariés. Cette évolution peut s'expliquer d'une part, par des facteurs endogènes au dispositif qui ont de toute évidence contribué favorablement à cette augmentation, tels la simplification des démarches administratives lors de la demande de financement ou le basculement des modalités de financement des activités de formation continue vers la bonification. D'autre part, comme évoqué précédemment, elle peut être due à l'évolution économique du pays, qui connaît dès les années 2008-09 une grave crise financière et immobilière dévastatrice en termes d'emplois influant sur le profil des entreprises recourant au dispositif de demande de formation. En effet, avec la crise, la taille des entreprises se réduit impactant de la sorte les statistiques produites par la FTFE.

À noter, toutefois, que malgré l'augmentation du nombre d'entreprises ayant recours au dispositif de demande de formation, une proportion toujours plus importante de ces firmes ne parvient pas au terme du processus de formation souhaitée, comme illustré dans le graphique qui suit.

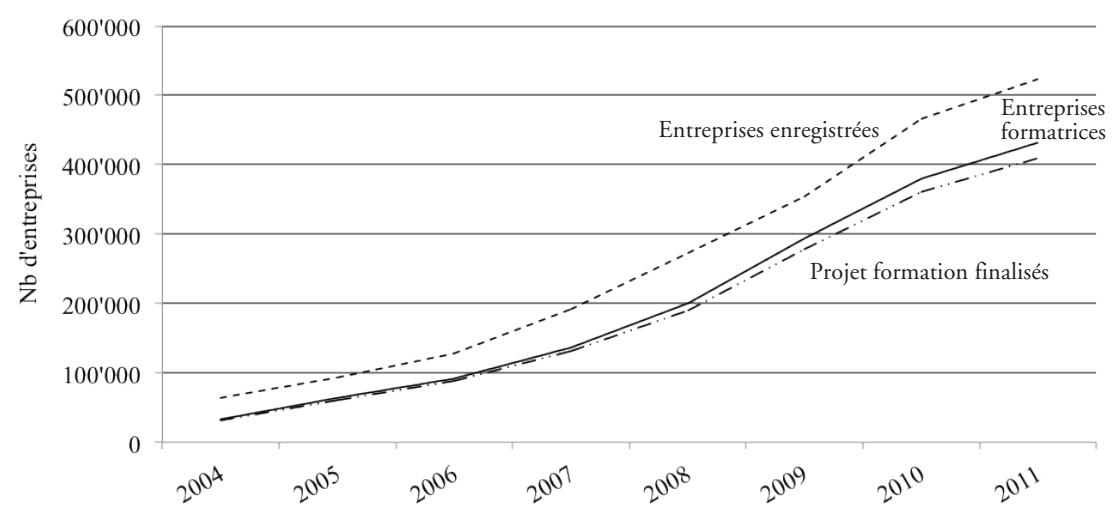

Graphique 3. Entreprises ayant mobilisé le dispositif de demande de formation de 2004 à 2011, en chiffres absolus

Source: auteure, calculé sur la base des données statistiques fournies par la FTFE

À cela plusieurs explications évoquées dans le rapport GAPEF-GIPE: tout d'abord, la difficulté d'accéder au financement en raison des conditions posées par le dispositif, à savoir, la procédure de demande de bonification jugée trop 
rigide car elle exclut de la demande des projets de formation novateurs qui seraient pourtant susceptibles de répondre aux besoins spécifiques des entreprises, ainsi que les montants alloués par le dispositif qui se révèlent insuffisants pour couvrir les coûts réels encourus par les employeurs. Les PME sont les premières pénalisées en raison de leur difficulté à assumer les coûts indirects engendrés par les activités de formation qui ne sont pas entièrement pris en charge par le dispositif de financement. À cela s'ajoute la difficulté rencontrée par les petites entités à définir leurs besoins de formation et à cerner les possibilités véritablement offertes par le dispositif. Les mesures d'accompagnement pour définir les besoins de formation et mener jusqu'à leur terme les procédures de demande de financement, jugées insuffisantes par les experts interviewés dans le cadre de l'enquête menée par les équipes GAPEF-GIPE, ainsi que la lourdeur administrative qui les accompagne sont pointées du doigt et lèsent les petites structures. Le coût d'opportunité reste, par ailleurs, un frein important malgré sa prise en charge par le dispositif car non seulement il est difficile à quantifier mais la compensation financière proposée ne suffit pas à couvrir tous les tracas causés par l'absence des salariés en formation.

Soulignons le manque d'attrait du dispositif auprès des entreprises ne percevant pas, ou jugeant insuffisants, les effets produits par la formation sur les résultats économiques de la firme. Il est à craindre que sans une culture d'entreprise forte, favorable à la formation, sans un positionnement de la formation continue comme outil stratégique dans la gestion à long terme des affaires entrepreneuriales et de moyens humains et financiers adéquats pour assumer les implications financières et organisationnelles induites par l'absence des salariés en formation, aucune mesure ne soit suffisante pour insuffler un véritable élan des actifs occupés vers la formation.

\section{L'instrumentalisation de la formation par les entreprises}

Le troisième point de réflexion concerne la problématique de l'instrumentalisation de la formation par l'employeur que le dispositif peine à déjouer. Ce phénomène touche particulièrement les publics peu ou pas qualifiés comme le confirment, par exemple, les études portant sur le contexte français (Dubois \& Melnik-Olive, 2017).

Ce phénomène reste très marqué dans le contexte espagnol malgré la mise en place d'un système de financement de la formation continue orienté vers la demande sensé offrir davantage de latitude aux salariés dans leur choix de formation. Cette instrumentalisation de la formation continue se révèle particulièrement à l'échelle des PIF, mesure pourtant prévue pour la contourner, puisqu'il ressort sans surprise de l'analyse que les personnes mobilisant cette option suivent les formations qui leur permettent de se maintenir en emploi. Ils orientent donc leur choix en fonction non seulement du profil de compétences recherché par l'employeur mais aussi des modalités de formation (type de formation, intensité, horaire de formation, etc.) susceptibles de coller au mieux 
aux «sacrifices» que l'employeur est prêt à consentir pour former ses salariés. Sauf à compter sur un employeur conciliant, les salariés se plient aux desiderata de leur entreprise en matière de formation. Phénomène également constaté notamment dans le contexte français (Dubois \& Melnik-Olive, 2017).

La nature de la formation choisie

Le quatrième point qu'il convient d'évoquer porte sur la nature de la formation mobilisée par les entreprises espagnoles. On observe tout d'abord une augmentation du taux de participation des actifs occupés au dispositif de demande de formation ( $48 \%$ en $2004,78 \%$ en 2010 ), à visée spécifique, au détriment du recours à l'offre de formation générale entre 2004 et 2010. Cette évolution est moins marquée s'agissant des femmes (1,31\% en $2004,6,39 \%$ en 2010$)$, des seniors de plus de quarante-cinq ans $(0,64 \%$ en $2004,3,6 \%$ en 2010$)$ et des salariés les moins qualifiés ( $0,66 \%$ en $2004,3,14 \%$ en 2010$)$. Le même scénario s'observe en termes de volume total d'heures de formation consommées. Jusqu'en 2004 , seuls $30 \%$ du volume total d'heures de formation profitent aux usagers du dispositif de demande de formation. Ce taux passe à $60 \%$ dès 2010 . Ce glissement révèle notamment le souci des entreprises de recourir à une formation répondant spécifiquement à leurs besoins, en termes de contenu comme de modalités.

\section{Le financement}

Le cinquième point concerne la question de la pertinence des montants de financement des activités de formation continue alloués par le dispositif ainsi que celle de la part de cofinancement direct de ces activités exigé aux entreprises de plus de neuf salariés. Ces mesures, telles que prévues dans le dispositif espagnol, dissuadent certaines entreprises de recourir à la formation. En cause tout d'abord, les montants alloués par le dispositif jugés souvent insuffisants pour financer les activités de formation envisagées, en particulier la formation spécifique plus onéreuse, parce que taillée sur mesure et ne permettant donc pas de bénéficier des économies d'échelle offertes par une formation standardisée. Cet aspect est fondamental lorsqu'on le met en résonance avec les mécanismes de la Théorie du capital humain (Becker, 1964) qui souligne l'importance d'investir en formation spécifique pour améliorer la productivité de l'entreprise. Dès lors, les entreprises recherchent ardemment des formations susceptibles de répondre à leurs besoins spécifiques.

À cela s'ajoute l'obligation pour les entreprises de cofinancer les activités de formation à hauteur d'un pourcentage établi en fonction de la taille de l'entreprise, elle-même définie en fonction du nombre de salariés qu'elle occupe. Cette obligation pose essentiellement deux problèmes que certaines firmes peinent à surmonter. Le premier est lié à la capacité financière de l'entreprise. En effet, certains employeurs ne disposent pas des liquidités nécessaires pour faire face à cette obligation de cofinancement les condamnant ainsi à renoncer au projet de formation envisagé pour leurs salariés. Le deuxième problème identifié est celui de faire reposer le pourcentage de cofinancement sur la taille de l'entreprise 
sachant que cet indicateur n'est pas révélateur de la capacité de financement d'une firme. En effet, le nombre de salariés n'est pas garant de la vitalité financière d'une entreprise.

Ces deux facteurs ont pour conséquence de détourner de la formation certains employeurs et contribuent à ce que l'on observe dans la configuration espagnole un sous-investissement des fonds publics dans la formation continue comme l'illustre le graphique suivant. En effet, le volume de financement public mobilisé par les entreprises est nettement inférieur au volume de financement public disponible.

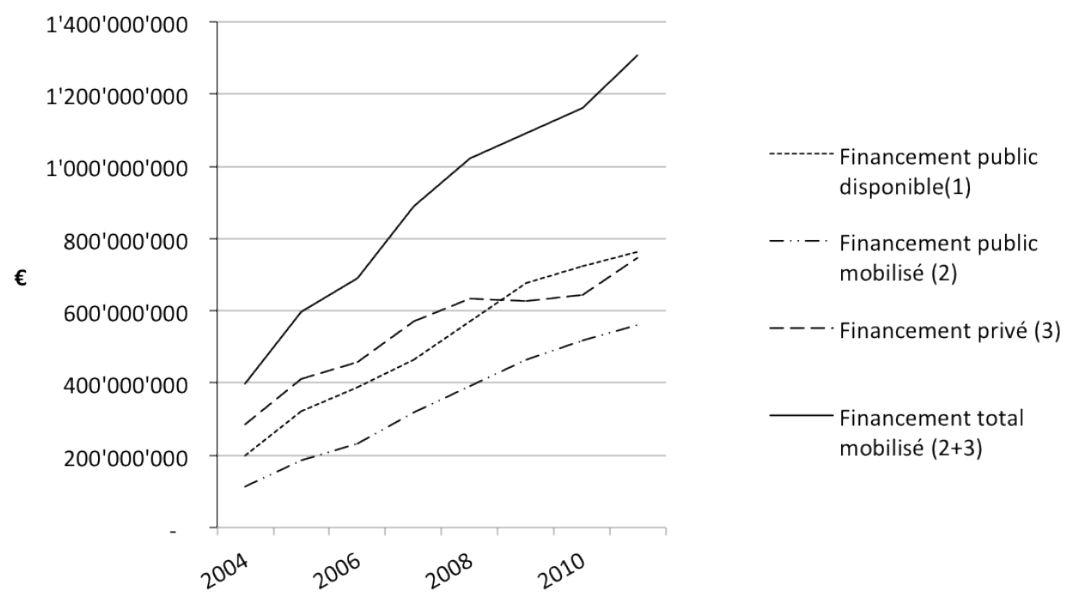

Graphique 4. Nature du financement de la formation continue de 2004 à 2010

Source: auteure, calculé sur la base des données statistiques fournies par la FTFE

L'obligation de cofinancement des activités de formation assure, en revanche, une certaine efficience économique au financement public dans la mesure où elle permet de contrecarrer de manière relativement efficace le risque de substitution d'un financement privé par un financement public tant redouté par les pouvoirs publics. En effet, le fait que le financement public ne puisse être utilisé que simultanément au financement privé est un garde-fou limitant la substitution des fonds. La question reste ouverte concernant les petites entités qui bien que petites peuvent avoir les moyens économiques d'assumer les coûts de la formation. Dans ce cas d'espèce, en établissant l'obligation de cofinancement sur la base du nombre de salariés occupés, les pouvoirs publics espagnols courent le risque de voir le financement public de la formation continue perdre de son efficience. 


\section{Les limites du financement public orienté vers la demande}

Le sixième point qu'il convient d'évoquer porte plus généralement sur les buts visés par les pouvoirs publics en mettant en place un financement public de la formation continue et sur la pertinence de son orientation vers la demande. Alors qu' une orientation de ce financement vers la demande laisse a priori présager une liberté d'accès à la formation continue à tout actif occupé car la décision de s'engager en formation ne dépend plus exclusivement de l'employeur, jusqu'alors bailleur de fonds, l'analyse du dispositif espagnol fait ressortir une mécanique différente.

En effet, l'employeur reste le moteur dans l'engagement d'un salarié en formation que le financement s'oriente vers l'offre ou vers la demande. À cela une raison qui tient à la finalité même de la formation envisagée ou entreprise. En se formant, l'individu cherche avant tout à maintenir ou à développer son employabilité, qu'elle soit interne ou externe. Pour cela, il opte pour la formation qui lui permette de développer les compétences recherchées par le marché du travail. L'employeur, qu'il soit présent, ou futur d'ailleurs, téléguide en quelque sorte l'accès à la formation continue. Il décide directement ou indirectement de qui suit la formation et quel type de formation sera mobilisé. L'employeur orientera ses choix en privilégiant la recherche de rentabilité économique. Restent ainsi sur le carreau les publics dont les perspectives de rentabilisation de l'investissement en formation continue sont jugées moindres, à savoir les travailleurs peu qualifiés, les seniors de plus de quarante-cinq ans et les salariés du marché du travail secondaire. Sont également écartés de la formation les actifs occupés ne se sentant pas concernés par la formation que ce soit par manque de motivation, de perspectives professionnelles ou parce qu'ils ne se sentent pas capables ou en mesure de parvenir à se former. Ces constatations invalident l'hypothèse selon laquelle l'orientation du financement de la formation continue vers la demande concèderait une liberté d'accès à la formation continue à tout actif occupé car non seulement l'employeur influence quoi qu'il en soit directement ou indirectement les choix en matière de formation mais l'accès à la formation continue étant multifactoriel, il ne dépend donc pas seulement d'une question de financement.

\section{Conclusion}

Au vu de ce qui précède, le dispositif de cofinancement tripartite de la formation continue orienté vers la demande en vigueur en Espagne de 2004 à 2011 apporte une manne de financement bienvenue aux entreprises et contribue à améliorer de manière générale l'accès à la formation continue des actifs occupés espagnols. En ce sens, le dispositif a un effet incitatif à investir en formation. Son efficacité est toutefois égratignée par le fait que cette augmentation du recours à la formation continue reste moins marquée pour les publics habituellement lésés dans cet 
accès, en l'occurrence, les salariés peu qualifiés, les seniors et ceux insérés dans le marché du travail secondaire.

En cause notamment la difficulté rencontrée par le dispositif à contrecarrer l'impact du coût d'opportunité sur la décision des entreprises d'investir en formation, en particulier lorsqu'il s'agit de PME dont les moyens financiers et humains retreints représentent un frein d'accès à la formation continue. Les montants de financement alloués par le dispositif s'avèrent insuffisants pour compenser les coûts indirects induits par l'absence des collaborateurs en formation. Ce d'autant que certains de ces coûts ne sont pas quantifiables monétairement mais pèsent néanmoins lourdement dans la balance des comptes.

Par ailleurs, l'obligation de cofinancer les activités de formation par les employeurs a pour effet de limiter l'attrait du dispositif. Certaines PME notamment sont contraintes de renoncer à l'investissement par manque de moyens financiers. Ce cofinancement limite en revanche le risque d'effet de substitution des fonds privés par des fonds publics ce qui contribue à l'efficience de l'investissement public en matière de formation continue.

Finalement, le dispositif espagnol ne parvient pas non plus à déjouer complètement l'instrumentalisation de la formation par l'employeur. En posant ses exigences, ce dernier détient indirectement un pouvoir décisionnel sur le choix de la formation. Lorsqu'il lance à son collaborateur une injonction à se former, mettant en péril l'employabilité interne de ce dernier s'il n'obtempère pas, aucune marge de manœuvre n'est laissée à l'individu. Le salarié est alors mû par une motivation extrinsèque le conduisant à se former conformément aux souhaits émis par l'employeur qui n'aura de cesse de prescrire le profil de compétences à acquérir et les modalités de la formation qui lui conviennent. Dans ce cas de figure, l'accès à la formation se fait par défaut, sans que le salarié ne maîtrise lui-même l'entier du processus. Il convient alors de questionner l'ampleur de la responsabilité individuelle à se former. Il en résulte une implication passive du collaborateur dans l'activité de formation avec le risque que ne soit entravés à la fois le développement de ces compétences et le transfert de ces dernières dans son activité professionnelle. In fine la rentabilité économique de l'investissement en formation s'en trouve pénalisée ce qui contribue à détourner l'entreprise et l'individu de toute velléité de réinvestir en formation. Le cercle vicieux s'installe.

\section{Références bibliographiques}

Backes-Gellner, U. (2011). Analyse de l'efficacité de quelques instruments d'encouragement à la participation à la formation continue. Expertise de la Commission d'experts "Loi sur la formation continue " (art. 64a Cst.). Zürich: Université de Zürich-ISU-Institut für Strategie und Unernehmensökonomik.

Becker, G. S. (1964). Human capital: a theoretical and empirical analysis, with special reference to education. New York, NY: National Bureau of Economic Research.

Bernier, A. (2014). Les effets posibles de la formation sur la productivité: l'apport de données longitudinales. Relations industrielles, 69,(2), 366-387. 
CEDEFOP. (2015). On the way to 2010: data for vocational education and training policies. (Research Paper $n^{\circ} 45$. Country statistical overviews 2014 update). Luxembourg: Publications Office of the European Union.

Chéron, A. (2011). L'évolution de la formation professionnelle continue: une perspective internationale. Nice, France: EDHEC BUSINESS SCHOOL.

Cour des comptes de Genève. (2015). Evaluation du chèque annuel formation et de l'accès des adultes à un premier niveau de qualification (Rapport $\mathrm{N}^{\circ}$ 92). Cour des comptes. République et canton de Genève

CSRE. (2004). Vers un financement de la formation continue orienté vers la demande (Rapport de tendance $\left.\mathrm{n}^{\circ} 7\right)$. Aarau: Centre suisse de coordination pour la recherche en éducation.

Dautray, P. (2007). Economie de la connaissance, post fordisme et savoirs occupationnels en Espagne: une nouvelle donne. Savoirs, 3,(15), 105-120.

Dubois, J.-M., Marion-Vernoux, I. \& Noack, E. (2016). Le dispositif d'enquêtes Defis: un nouveau regard sur la formation en entreprise. Bref du Céreq, 344, 1-4.

Dubois, J.-M. \& Melnik-Olive, E. (2017). La formation en entreprise face à l'aspiration des salariés. Céreq Bref, 357, 1-4.

Fundación Tripartita para la Formación en el Empleo (FTFE). (2010). Evaluación de la iniciativa de bonificaciones de acciones de formación en las empresas. Ejecicios 2007 y 2008. Madrid: Madrid : Fundación Tripartita para la Formación en el empleo.

Hanhart, S. (2007). Les entreprises suisses: un investissement faible et sélectif dans la formation continue. Formation emploi, 100, 65-78.

Hansson, B. (2008). Job-Related Training and Benefits for Individuals. A Review of Evidence and Explanations. OECD Education Working Papers, 19, 1-48.

Lambert, M. \& Marion-Vernoux, I. (2014) (Eds). Quand la formation continue. Repères sur les pratiques de formation des employeurs et des salariés. Marseille Cédex 02: Céreq.

Ley 39/2010, de 22 de diciembre, de Presupuestos Generales del Estado para el año 2011.

Ley 17/2012, de 27 de diciembre de Presupuestos Generales del Estado para el año 2013.

OCDE. (2004). Cofinancer la formation tout au long de la vie: Vers une approche systémique. Paris: OCDE.

Orden TAS /2307/2007, Boletín Oficial del Estado (BOE), de 27 de julio.

Pichler, E. (1993). Cost-sharing of General and Specific Training with Depreciation of Human Capital. Economics of Education Review, 12,(2), 117-124.

Pineda-Herrero, P. (2007). La formación continua en España: Balance y retos de futuro. Relieve, 13,(1), 43-65.

Pineda, P. \& Sarramona, J. (2006). El nuevo modelo de formación continua en España: balance de un año de cambios. Revista de Educación, 341(Septiembre-diciembre), 705-736.

Real Decreto 395/2007, Boletin Oficial del Estado (BOE), de 23 de marzo.

Voirol-Rubido, I. (2017). Comment financer la formation continue en Suisse? Formation Emploi, 137, 15-32.

Wolter, S. C. \& Messer, D. (2009). Formation continue et bons de formation. Résulats d'une expérience menée sur le terrain. Berne: OFFT.

Woodhall, M. (2004). L'analyse coût-bénéfice dans la planification de l'éducation. Paris: UNESCO.

Mots-clés: Cofinancement de la formation continue, orienté vers la demande, Espagne, efficacité, équité d'accès 


\section{Ein Modell der Kofinanzierung von kundenorientierter Weiterbildung: das Spanische Beispiel}

\section{Zusammenfassung}

Dieser Artikel interessiert sich für die Wirkungen eines kundenorientierten Finanzierungsmodells im Bereich der Weiterbildung. Er analysiert die Qualität dieses spanischen Modells hinsichtlich dessen Wirksamkeit, des gleichberechtigten Zuganges und des Ansporns für spanische Arbeitskräfte, sich weiterzubilden. Anhand eines mixed-method Ansatzes (quantitativ-qualitativ) wird das Potential dieses Modells aufgezeigt, das in der Verbesserung des Zugangs zu Weiterbildung für spanische Arbeitskräfte zwischen 2004 und 2011 besteht. Gleichzeitig zeigt der Artikel die Schwierigkeit dieses Modells auf, die Instrumentalisierung im Bereich der Weiterbildung durch die Arbeitgeber zu verhindern. Zudem stellt der Einfluss der Kostenfrage, die möglicherweise eine kostenintensive Weiterbildung zum Vornherein degradiert, ein weiteres Problem dar.

Schlagworte: Kofinanzierung, Weiterbildung, Kundenorientierung, Spanien, Wirksamkeit, Chancengleichheit

\section{Un modello di cofinanziamento della formazione continua orientato verso il cliente: il caso spagnolo}

Riassunto

Questo articolo tratta degli effetti di un dispositivo di finanziamento della formazione continua orientato al cliente. In particolare esso affronta la qualità del dispositivo spagnolo in termini di efficacia, di equità di accesso e di incentivazione dei lavoratori attivi spagnoli ad impegnarsi in formazione. Sulla base di un'analisi di dati secondari che combinano una metodologia di natura quantitativa e qualitativa, si mette in evidenza il potenziale di tale dispositivo nell'incrementare l'accesso alla formazione dei lavoratori spagnoli tra il 2004 e il 2011. Emerge tuttavia la difficoltà del dispositivo a contrastare la strumentalizzazione della formazione da parte dei datori di lavoro ed a contenere l'impatto dei costi sulla possibilità di decidere a favore della formazione, aspetto che penalizza l'investimento stesso nella formazione continua.

Parole chiave: Cofinanziamento della formazione continua, orientata verso la domanda, Spagna, efficacia, equità di accesso 


\section{A Cofinancing Demand-Oriented Model of Continuous Vocational Training: the Spanish Case}

\section{Abstract}

This article aims to analyse the potential of a continuous vocational training (CVT) demand oriented financing system. Based on a secondary data analysis combining quantitative and qualitative methodologies, it analyses the quality of the Spanish system focusing on its efficiency, equity of access and potential to incentive employees to be trained between 2004 and 2011. The results suggest that the system has positive effects on Spanish workers' access to CVT. However, it cannot counter the training instrumentalization by employers, nor can it thwart the opportunity costs impact on the decision to invest in CVT which obviously penalises training investment.

Key words: CVT cofinancing, demand oriented, Spain, efficiency, equity of access 